\title{
Multiphase Catalytic Hydrogenation of $p$-Chloroacetophenone and Acetophenone. A Kinetic Study of the Reaction Selectivity toward the Reduction of Different Functional Groups
}

\author{
Pietro Tundo, ${ }^{1}$ Sergei Zinovyev, ${ }^{2}$ and A Ivise Perosa \\ D ipartimento di Scienze A mbientali, U niversità Ca' Foscari, D orsoduro 2137, 30123 Venezia, I taly
}

R eceived A pril 25, 2000; revised A ugust 4, 2000; accepted A ugust 31, 2000

\begin{abstract}
A multiphase catalytic system consisting of hydrocarbon solvent and aqueous phase, phase-transfer (PT) agent (A liquat 336), and supported Pt or Pd catalyst, bubbled with hydrogen at atmospheric pressure, affords the rapid and efficient hydrodechlorination and the selective reduction of the functional groups of $p$-chloroacetophenone and acetophenone at $50^{\circ} \mathrm{C}$ and in moderate reaction times. The reaction gives quantitative yields of reduction products with variable selectivity, which can be controlled by varying some simple reaction conditions. Herein both halogen removal and carbonyl group or phenyl ring reductions are discussed. The composition of the aqueous phase, the nature of supported catalysts, the effect of various inorganic anions, and other reaction conditions have been studied kinetically to estimate the mechanistic aspects of the reaction selectivity. E mphasis is placed on the development of a novel synthetic tool, which allows control over the selectivity in the reduction of the carbonyl group and the phenyl ring. A number of kinetic models and the corresponding integral rate expressions were used, coupled with the "simultaneous nonlinear least-squares fitting" method, to estimate the rate constants for the formation of each particular reaction component. The kinetic monitoring allowed the accurate analysis of the reaction selectivity. Some mechanistic conclusions were drawn for the reaction under study that suggest that "softer" reduction conditions are attained in the presence of a PT agent and the aqueous phase. (c) 2000 A cademic Press
\end{abstract}

Key Words: catalytic hydrogenation; multiphase conditions; Pt/C catalyst; phase-transfer catalysis; kinetic study.

\section{INTRODUCTION}

It has recently been reported that a new hydrodehalogenation (HDH) system, consisting of aqueous basic and organic phases, and a supported metal (Pt or Pd) catalyst coupled with a phase-transfer (PT) agent, affords the

\footnotetext{
${ }^{1}$ To whom correspondence should be addressed. Fax: +39 (041) 257 8620. E-mail: tundop@unive.it.

${ }^{2}$ O n leave from N.D. Z elinsky Institute of O rganic Chemistry of R ussian A cademy of Sciences, Leninsky pr. 47, M oscow 117913, R ussia.
}

rapid and efficient removal of chlorine and other halogens (1-3) as well as the reduction of other functional groups (3-5). The reaction gives quantitative yields of reduction products under extremely mild conditions: hydrogen at atmospheric pressure, $50^{\circ} \mathrm{C}$, and moderate reaction times, whereas in most other systems known for H D H in the presence of supported metal catalysts (e.g., the gaseous phase reaction under continuous flow), harsh temperature and pressure are required (6-9). M oreover, the supported catalyst under these conditions is easily poisoned by side products (e.g., $\mathrm{HCl})(7,10)$ and has short-lived activity. Examples of $\mathrm{HDH}$ with polymer-immobilized $\mathrm{R} h$ complexes are also known (11), but involve more complicated systems.

A peculiarity of the reaction is its broad scope: in particular, it has synthetic value, and it is a promising technique for the degradation of toxic chlorinated pollutants. From the synthetic standpoint, it is a versatile reduction technique for the preparation of valuable products, and it is conceivable that this may be done in an ecofriendly manner, starting from the selective removal of halogen from undesired chlorinated aromatics (12). There are, in fact, a variety of publications (13-16) on the catalytic heterogeneous hydrodechlorination (HDC) over supported (Pt, Pd, Ru, etc.) catalysts, aimed at the utilization of hazardous compounds such as polychlorinated biphenyls (PCB s) (2), pesticides (14), chemical weapons $(15,16)$, and other polychlorinated aliphatics/aromatics, which have been produced in huge amounts on the industrial scale.

M ultiphase HDH can be also used as a mild method for the detoxification of hazardous chlorinated aromatic pollutants and of persistent organic pollutants such as polychlorinated dibenzo-p-dioxins (PCDD s), polychlorinated dibenzofurans (PCD Fs), and PCB s (2). I t can therefore become a valuable technique for the detoxification of polychlorinated pollutants present in organic wastes, incinerator gases, contaminated materials, and environmental matrices.

It has also been shown that under these new multiphase conditions, which were originally developed for the dehalogenation of various haloaromatics, the selective reduction 
$(4,5)$ of carbonyl or hydroxyl group, or phenyl ring reduction, may take place as well. Y et, it has been shown (4) that by varying simple reaction conditions one can switch the selectivity of the reduction toward more or less reduced products or control the selectivity in the parallel reduction steps, i.e., in the reduction of the carbonyl versus the aromatic ring. The main factors, which have been proven to have such an influence, are the composition of the aqueous phase, and the nature and the ratios of the supported metal catalyst and of the PT agent (4). A Ithough the system may appear complicated due to the number of different phases present (we can count five: aqueous, organic, supported catalyst, PT agent which coats the catalyst and forms a third liquid phase, and gaseous $\mathrm{H}_{2}$ ), and although interpretation of how each variable comes into play is difficult, the reaction itself is carried out easily even on preparative scales.

The complete mechanism is still under investigation; however, evidence suggests that the PT agent forms a third liquid phase over the surface of the supported metal catalyst, thereby modifying the chemical environment on the solid catalyst surface. A ccording to a model mechanistic scheme published elsewhere (5), the reaction takes place in this third liquid phase and the selectivity is affected by the different geometry of substrate adsorption on the catalyst surface. Therefore, the selective reduction of different functional groups should be affected by the nature of the third liquid phase, and such factors as the nature of PT agents, the additions of various anions, and the $\mathrm{pH}$ of the aqueous phase appear to be critical.

This work is aimed at the investigation of the reaction selectivity, by measuring the observed kinetic constants of each step. The kinetic data analysis and the detailed monitoring of the different reaction conditions lead to accurate conclusions about the peculiarities of the multiphase conditions studied. The examples chosen are $p$ chloroacetophenone and aceto phenone; the latter has been studied in more detail since we were interested in the selectivity of the reduction of functional groups other than chlorine, and especially in the selectivity toward the reduction of the aromatic ring, which is unfavorable under the usual catalytic conditions (6-9).

\section{METHODS}

A II reagents and solvents were ACS grade and were used without further purification. The $5 \% \mathrm{Pt} / \mathrm{C}$ was from Fluka, A rt. N o. 80982; its surface area was $700-800 \mathrm{~m}^{2} / \mathrm{g}$. The $10 \% \mathrm{Pd} / \mathrm{C}$ was from A ldrich, A rt. N o. 20, 569-9. G C analyses were performed on a Varian GC 3400 using a fused silica capillary column "Chrompack CP Sil 8 CB" (30 $\mathrm{m} \times 0.32 \mathrm{~mm}$ ) with $95 \%$ dimethyl- $5 \%$ diphenyl polysiloxane as the stationary liquid phase (film thickness $0.25 \mu \mathrm{m}$ ). G C/M S analyses were performed on a H P 5971 mass de- tector coupled to a H P 5890 gas chromatograph fitted with a 30-m $\times 0.25-\mathrm{mm}$ D B 5 capillary column.

G eneral Procedure for $p-C$ hloroacetophenone and A cetophenone R eduction

A $25-\mathrm{ml}$ three-necked round-bottomed flask thermostated at the reaction temperature $\left(50 \pm 0.1^{\circ} \mathrm{C}\right)$, connected with a system for the addition of hydrogen and with a waterjacketed condenser, was employed for the reaction. If not otherwise indicated, the reactor was loaded with a mixture of an aqueous phase $(5.7 \mathrm{ml}$; for the composition of the aqueous phase see the conditions indicated in the tables), $5 \%$ Pt/C ( $0.0837 \mathrm{~g} ; 0.021 \mathrm{mmol}$ of Pt), A liquat 336 (tricaprylmethylammonium chloride, $0.103 \mathrm{~g}, 0.26 \mathrm{mmol}$ ), $0.7 \mathrm{mmol}$ of substrate ( $p$-chloroacetophenone or acetophenone), and n-decane as the internal standard $(0.0407 \mathrm{~g}$, $0.3 \mathrm{mmol}$ ). The organic phase was adjusted to $10 \mathrm{ml}$ by adding the corresponding amount of isooctane. The reaction mixture was magnetically stirred at $1000 \mathrm{rpm}$. Hydrogen was bubbled at atmospheric pressure into the organic phase at about $5 \mathrm{ml} / \mathrm{min}$.

\section{Sampling P rocedure and G C A nalyses}

The samples were collected from the organic phase during the reaction at time intervals. The first one was taken just after the reagents had been loaded and it was accounted for as zero time. A bout $20 \mu \mathrm{l}$ of the reaction mixture was taken for each sample, which was then diluted with ethyl ether up to 1-2 $\mathrm{ml}$. The resulting sample was then shaken with silica and filtered with cotton wool to eliminate the impurities of A liquat 336 and $\mathrm{Pt} / \mathrm{C}$ particles.

$D$ etermination of the concentration of the reaction components was performed using the internal standard technique. For this reason, a number of calibration coefficients were calculated for each reaction component by analyzing the binary mixtures of these compounds with $n$-decane. The calibration coefficients were calculated by plotting the ratios of the component-decane $\mathrm{GC}$ peak areas versus the ratios of their known concentrations in the corresponding binary mixtures. Taking into account the known concentration of the internal standard, the calibration coefficients $\left(k_{\text {cal }}\right)$ for $p$-chloroacetophenone, acetophenone, phenyl ethanol, cyclohexyl ethanol, acetyl cyclohexane, ethyl benzene, ethyl cyclohexane, p-chlorophenylethanol, and pchloroethylbenzene were calculated as $0.046,0.039,0.038$, $0.040,0.037,0.026,0.035,0.046$, and 0.031 , respectively. The following expression was used for the calculation of component concentrations: $C_{c o m p}=k_{\text {cal }}\left(S_{\text {comp }} / S_{\text {stand }}\right)$, where $S_{\text {comp }}$ and $S_{\text {stand }}$ are the GC peak area units for the analyzed compound and for the internal standard, respectively. The results were successfully tested for reproducibility. The systematic error for reproduction, reflected in the determination of rate constants, was no higher than $10 \%$. The 
first-order rate constants were obtained by using a fitting technique described herein.

\section{RESULTS AND DISCUSSION}

\section{O bserved R ate Constants and A dsorption Contribution}

The investigation of the hydrogenation reaction under multiphase conditions has been carried out using $p$ chloroacetophenone and acetophenone as model compounds. Since the reaction pathways usually differ from one system to another, it is helpful to use a scheme which shows all the possible reaction pathways (Scheme 1) for both $p$ chloroacetophenone and acetophenone reductions. Steps which account for the reduction of the aromatic ring leaving chlorine did not occur in any system and therefore are not included.

Each reaction step in this scheme has its own rate constant. The observed kinetics are rather complicated due to there being a number of intermediates, but can be described using the developed mathematical approach if each step is assumed to be pseudo-first-order (see the following paragraph).

Sets of calculated first-order rate constants for each particular experiment have been obtained using the "simultaneous nonlinear least-squares fitting" technique and are listed in tables using the definitions corresponding to Scheme $1\left(k_{n}{ }^{\prime \prime}, n=1,2, \ldots, 7\right)$. The rate constants obtained remain the observed ones since adsorption coefficients are not considered. Differential adsorption of all the reaction intermediates and reagents over the supported catalyst is an important factor which contributes to the observed rate constants. A ccording to the Langmuir-H inshelwood approach, exemplified by B eranek (17) for some complex kinetic schemes in heterogeneous catalysis, the reaction rate can be expressed as

$$
\text { Cl- }
$$

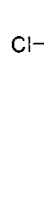

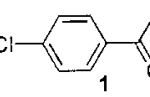
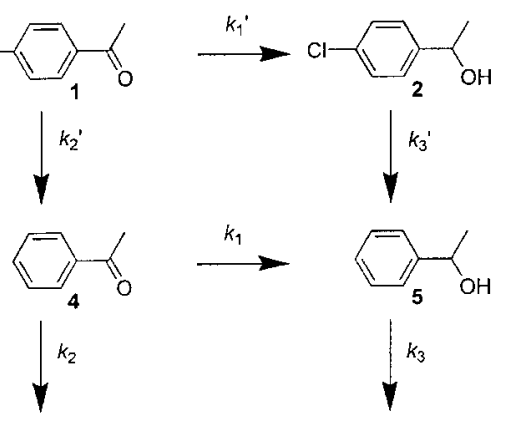
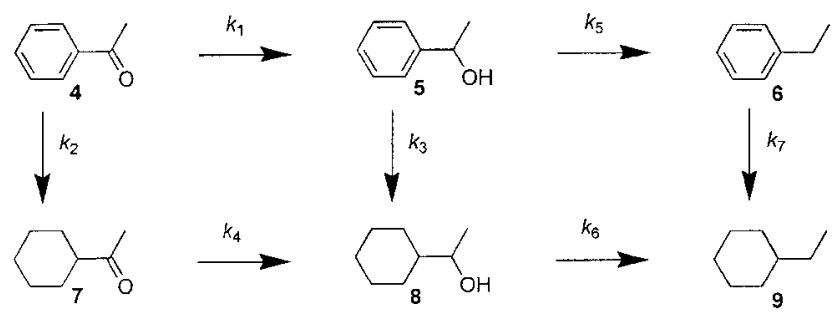

$$
r_{i j}=\frac{k_{i j} K_{i} P_{i} P_{\mathrm{H}_{2}}^{n(m)}}{\left(1+\sum_{i} K_{i} P_{i}\right)^{b}},
$$

SCHE ME 1. Possible reaction pathways for the catalytic hydrogenation of $p$-chloroacetophenone and acetophenone. where $k_{i j}$ is the intrinsic rate constant for the $i j$-th step, $\mathrm{P}_{\mathrm{i}}$ and $\mathrm{P}_{\mathrm{H}_{2}}$ are the partial pressures of the $\mathrm{i}$-th reactant and hydrogen, respectively, the term in the denominator is the surface term, and $\mathrm{K}_{\mathrm{i}}$ is the equilibrium adsorption coefficient. The surface term will cancel since the surface is identical for all species, and assuming that the partial pressure of hydrogen is constant, then the observed relative rate constant $k_{i j}{ }^{\prime}$ (if the usual first-order approach is used) will be represented as a product of the intrinsic rate constant $k_{i j}$ and the equilibrium adsorption constant $\mathrm{K}_{i}$. This approach has been used for the similar case of heterogeneous H D H in the vapor phase (9).

However, even if the adsorption coefficients do affect the observed rate constants, we still assume that the adsorption corrections can be omitted: in fact, the present kinetic model is mainly directed toward the appraisal of the actual reaction selectivity, and it is applicable to this end. The intrinsic rate constants cannot be easily calculated since the determination of the adsorption coefficients is troublesome; under our multiphase conditions the adsorption terms ( $K_{i}$ and the surface term) appear to be different for each intermediate from one system to another, since the catalyst surface is modified by the components, according to the reported mechanism (5). M oreover, the multiphase system seems too complicated for the usual adsorption estimation (17), because the reactants do not directly sorb on the catalyst surface, but transfer to the different environment of the third liquid layer.

B esides affecting rate constants, adsorption may affect the reaction kinetics as well, by causing deviation of the observed reaction profiles from the model first-order kinetics. The error due to this effect and to side products can be corrected by verifying the mass balance of the reaction. In our case, the reaction components can be accounted for within $10 \%$, implying that the reaction kinetics can still be accurately processed using the first-order approach.

\section{$K$ inetic M odels and $D$ ata A nalysis}

A number of kinetic models and the corresponding mathematical equations have been developed in order to perform the computational integral kinetic analysis of the data obtained. The mathematical approach for Scheme 1 appears to be too complicated to perform an accurate estimation of the experimental data. M oreover, in all the cases studied, depending on the conditions employed, only some particular reaction steps take place, and so only the intermediates corresponding to these steps have been analyzed. Therefore, the kinetic approach employed for the particular cases makes it possible to reduce the overall reaction scheme to a number of less complicated ones. A II the reaction pathways, which take place in the reductions of acetophenone and p-chloroacetophenone, can be divided into seven different cases; for each of them the corresponding mathematical approach has been developed. The global mechanistic scheme is presented in Scheme 7 of Table 1. 
TABLE 1

Model Mechanistic Schemes U sed for the Interpretation of Reaction Pathways

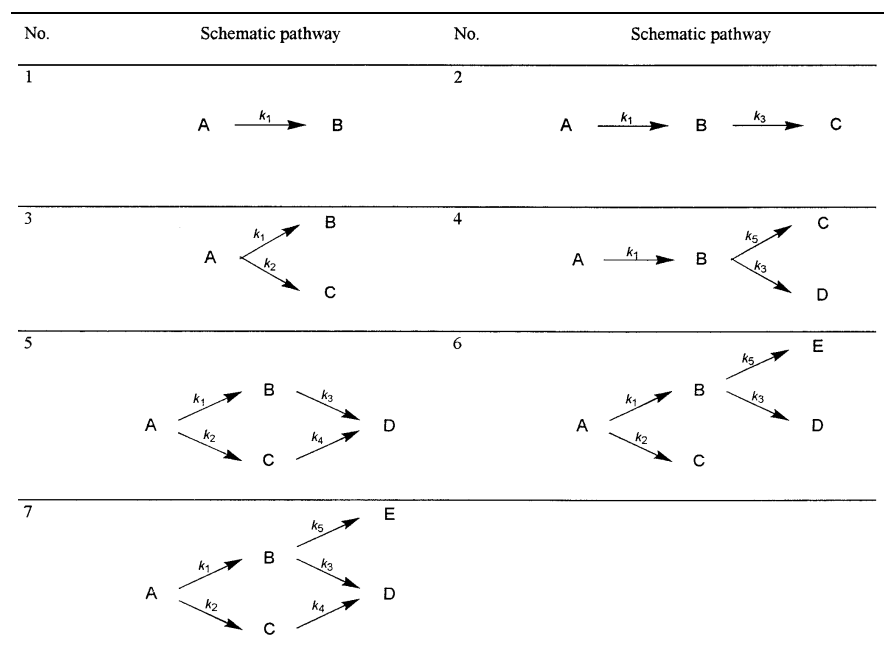

The definitions used for compounds ( $, B, C, D, E)$ and for rate constants $\left(k_{1}, k_{2}, k_{3}, k_{4}, k_{5}\right)$ in Table 1 represent the formal approach and do not necessarily correspond to those in Scheme 1. These definitions can be substituted for ones which are used for the actual constants by simply matching the geometry of the reaction pathway with the corresponding one in Table 1.

Based on the model mechanistic schemes presented in Table 1, a number of integral kinetic expressions can be written. E ach of these expressions accounts for a certain reaction intermediate, product, or substrate. It is obvious that the most complex model scheme (entry 7, Table 1) can be reduced to all the other cases (entries 1-6) by eliminating certain reaction steps. The same is true for the mathematical expressions if the corresponding rate constants are set to zero. The integral rate expressions, which represent the concentration dependencies of $A, B, C, D$, and $E$ for entry 7 , are derived from the following system of differential rate expressions:

$$
\begin{gathered}
\frac{d[\mathrm{~A}]}{d t}=-\left(k_{1}+k_{2}\right)[\mathrm{A}], \quad \frac{d[\mathrm{~B}]}{d t}=k_{1}[\mathrm{~A}]-\left(k_{3}+k_{5}\right)[\mathrm{B}], \\
\frac{d[\mathrm{C}]}{d t}=k_{2}[\mathrm{~A}]-k_{4}[\mathrm{C}], \quad \frac{d[\mathrm{D}]}{d t}=k_{3}[\mathrm{~B}]+k_{4}[\mathrm{C}], \\
\frac{d[\mathrm{E}]}{d t}=k_{5}[\mathrm{~B}] .
\end{gathered}
$$

By setting the initial conditions $[B],[C],[D],[E]=$ $0,[A]=[A]_{0}$ at $t=0$, and the mass balance $[A]_{0}=$ $[A]+[B]+[C]+[D]+[E]$, the solution for this system is the following set of integral rate expressions:

$$
\begin{aligned}
{[\mathrm{A}]=} & {[\mathrm{A}]_{0} e^{-\left(k_{1}+k_{2}\right) t} } \\
{[\mathrm{~B}]=} & {[\mathrm{A}]_{0} \frac{k_{1}}{k_{5}+k_{3}-K_{2}-k_{1}}\left(e^{-\left(k_{1}+k_{2}\right) t}-e^{-\left(k_{3}+k_{5}\right) t}\right) } \\
{[\mathrm{C}]=} & {[\mathrm{A}]_{0} \frac{k_{2}}{k_{4}-k_{1}-k_{2}}\left(e^{-\left(k_{1}+k_{2}\right) t}-e^{-k_{4} t}\right) } \\
{[\mathrm{D}]=} & \frac{1}{k_{1}+k_{2}}\left(k_{2}+\frac{k_{1} k_{3}}{k_{3}+k_{5}}\right)[\mathrm{A}]_{0} \\
& \times\left[1-\frac{1}{k_{5}+k_{3}-k_{2}-K_{1}}\left(e^{-\left(k_{1}+k_{2}\right) t}-e^{-\left(k_{3}+k_{5}\right) t}\right)\right. \\
& \left.-\frac{k_{2}}{k_{4}-k_{2}-k_{1}}\left(e^{-\left(k_{1}+k_{2}\right) t}-e^{-k_{4} t}\right)-e^{-\left(k_{1}+k_{2}\right) t}\right] \\
{[\mathrm{E}]=} & \frac{k_{1}}{k_{1}+k_{2}} \frac{k_{5}}{k_{3}+k_{5}}[\mathrm{~A}]_{0}\left[1-\frac{1}{k_{5}+k_{3}-k_{2}-k_{1}}\right. \\
& \left.\times\left(\left(k_{3}+k_{5}\right) e^{-\left(k_{1}+k_{2}\right) t}-\left(k_{1}+k_{2}\right) e^{-\left(k_{3}+k_{5}\right) t}\right)\right]
\end{aligned}
$$

These expressions, which are derived for the most complex mechanistic scheme (entry 7 in Table 1), can then be easily reduced to ones corresponding to all the other applied cases.

To process the experimental data, the "simultaneousnonlinear least-squares fitting" technique has been performed by means of the " $\mathrm{M}$ icroCal O rigin v. 5.0 PC software using the "simplex" iterations method. This technique allows a number of integral rate expressions (e.g., E qs. [1]-[5]), describing the formation (decomposition) of all the reaction components (e.g., A -E) in each particular experiment, to be used together to process all the corresponding data sets (the sets of experimental data on the component concentrations) simultaneously.

The simultaneous curve fitting appears to be an accurate technique for the analysis of complex reaction schemes. For example, it allows one to consider such complex expressions as [4] and [5], which contain four or five parameters and therefore are not likely to be applied separately. The simultaneous fitting technique affords a set of rate constants for each experiment by minimizing the approximation errors with respect to the formation of each component, which depends on these constants. A n example of the "nonlinear least-squares fitting" procedure is presented in Fig. 1.

Sets of first-order rate constants obtained for each experiment and the corresponding standard approximation errors are presented in Tables 2-5 using the models of Table 1 . The choice of the model was based on the preliminary appraisal of the component kinetic profiles (18). To understand which model has been used, it is enough to examine which constants are presented in the tables. The standard errors reported rarely exceed $20 \%$, which is quite acceptable for the integral kinetic analysis. 


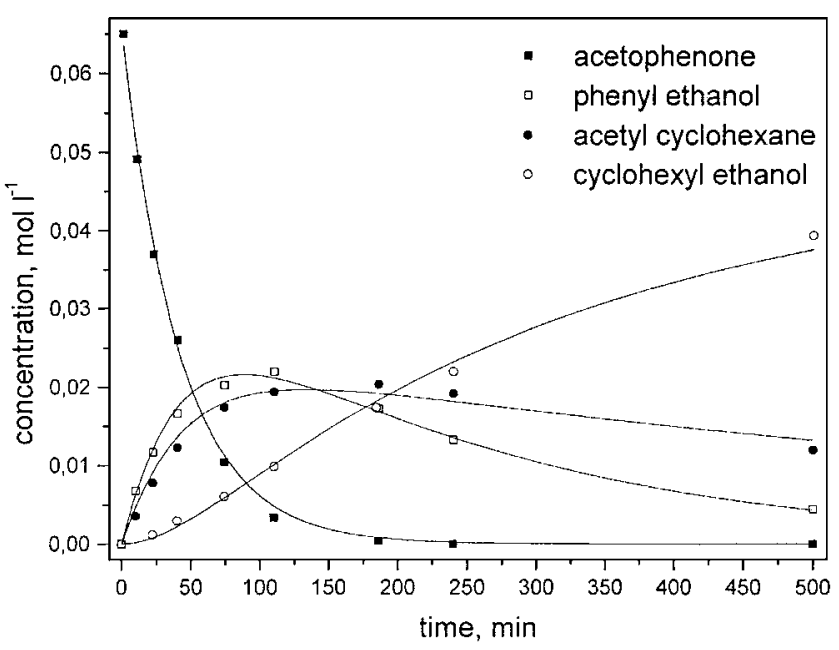

FIG . 1. "N onlinear least-squares fitting" procedure on the example of hydrogenation of acetophenone on $\mathrm{Pt} / \mathrm{C}$ with $\mathrm{A}$ liquat 336 and pure water (Table 3, entry 2). The kinetic model employed is that of entry 5 in Table 1. D ots and curves represent the experimental data and the corresponding theoretical integral rate dependencies, respectively.

When any of the intermediates or products are formed in negligibly small amounts (e.g., ethyl benzene and ethyl cyclohexane in Scheme 1), their exclusion from the kinetic scheme is warranted, as it simplifies the mathematical model, and it reduces the error on the other constants. M oreover, since the rate constants of their formation cannot be accurately measured, only the initial rate of formation is estimated by calculating the $\operatorname{tg} \alpha$ at time $=0$. The same linear approximation for the initial rate of formation was used for the products of very slow reaction steps.

$\mathrm{H}$ ydrogenation of $\mathrm{p}$-Chloroacetophenone without $\mathrm{B}$ ase and without PT A gent

p-Chloroacetophenone can be easily dechlorinated and reduced to ethyl cyclohexane with $\mathrm{H}_{2}$ and $\mathrm{Pt} / \mathrm{C}$ in a biphase aqueous-organic system, even in the absence of A liquat 336 and base (Table 2, entry 1 ). The reaction proceeds exclusively through the $\mathbf{1} \rightarrow \mathbf{2} \rightarrow \mathbf{3} \rightarrow \mathbf{6} \rightarrow \mathbf{9}$ pathway, affording ethyl cyclohexane (9) as a major product (90\%) with a comparatively high reaction rate. Small amounts of dechlorinated ketone (4) and alcohol (5) have been detected, which are formed with relatively low rates $\left(\mathrm{k}_{2}^{\prime} \ll\right.$ $\left.\mathrm{k}_{1}{ }^{\prime}, \mathrm{k}_{3}{ }^{\prime} \ll \mathrm{k}_{4}{ }^{\prime}\right)$ and readily give acetyl cyclohexane (7) and cyclohexyl ethanol, the latter in only $5-7 \%$ yield.

The results suggest that under these conditions chlorine is not likely to be removed from the aromatic ring before the other functional groups are reduced. The prohibition of the $\mathbf{8} \rightarrow \mathbf{9}$ step is also interesting and may result from the effect of the acidic medium, since the $\mathrm{HCl}$ released during the dechlorination may cause leaching processes (7) and inhibition of the activation of $\mathrm{H}_{2}$ on the catalyst (10), or from competing adsorption of aromatic intermediates on the catalyst. Seemingly, the aliphatic (cyclohexyl) derivatives are not likely to undergo reduction in the presence of aqueous acid. This observation is also supported by the hydrogenation of acetophenone in the presence of aqueous acid and $\mathrm{A}$ liquat 336 (Table 3).

$\mathrm{H}$ ydrogenation of $\mathrm{p}-\mathrm{C}$ hloroacetophenone with

\section{A liquat 336 and B ase}

In the presence of A liquat 336 and of $1 \%$ aqueous $\mathrm{KOH}$ solution, the hydrogenation of $\mathrm{p}$-chloroacetophenone proceeds with reversed selectivity (Table 2, entry 2 ). Chlorine is the first functional group to be reduced, so no formation of $\mathbf{2}$ and $\mathbf{3}$ is observed. The reaction pathway follows the $\mathbf{1} \rightarrow \mathbf{4} \rightarrow(\mathbf{7}+\mathbf{5})$ order, with the final selectivity toward the formation of $\mathbf{5}$. O nly negligible rates for the formation of $\mathbf{6}$, $\mathbf{8}$, and $\mathbf{9}$ have been observed. U nder these multiphase conditions, there is a high consecutive selectivity toward acetophenone (4) because the rate for the removal of chlorine $(\mathbf{1} \rightarrow \mathbf{4})$ is much higher than those of all subsequent reaction steps $(\mathbf{4} \rightarrow(\mathbf{7}+\mathbf{5}))$. This same effect has been previously observed for p-chloropropiophenone which, in the absence

TABLE 2

First-Order $\mathrm{R}$ ate $\mathrm{C}$ onstants for the Hydrogenation of $p$-Chloroacetophenone over $5 \% \mathrm{Pt} / \mathrm{C}$

\begin{tabular}{|c|c|c|c|c|c|c|c|c|c|c|c|}
\hline \multirow[b]{2}{*}{ No. } & \multicolumn{10}{|c|}{ First-order constants $\times 10^{3}\left(\mathrm{~s}^{-1}\right)$} & \multirow{2}{*}{$\begin{array}{l}\text { Products } \\
\text { molar ratio }\end{array}$} \\
\hline & $k_{1}$ & $k_{2}$ & $\mathrm{k}_{3}, \mathrm{k}_{4}, \mathrm{k}_{5}$ & $\mathrm{k}_{6}$ & $\mathrm{k}_{7}$ & $k_{1}^{\prime}$ & $k_{2}^{\prime}$ & $k_{3}^{\prime}$ & $k_{4}^{\prime}$ & $k_{5}^{\prime}$ & \\
\hline $1^{a}$ & $-^{c}$ & $--^{c}$ & $--^{c}$ & 0 & $0.58 \pm 0.13$ & $1.45 \pm 0.18$ & $-{ }^{d}$ & $--^{d}$ & $-^{\mathrm{e}}$ & $2.10 \pm 0.38$ & $1 / 15(\mathbf{8} / \mathbf{9})^{f}$ \\
\hline $2^{b}$ & $0.17 \pm 0.02$ & $0.02 \pm 0.00$ & 0 & 0 & 0 & 0 & $5.68 \pm 0.85$ & 0 & 0 & 0 & $1 / 10(\mathbf{7 / 5})^{g}$ \\
\hline
\end{tabular}

\footnotetext{
a $\mathrm{N}$ o A liquat 336 in the presence of water.

${ }^{\mathrm{b}} \mathrm{A}$ liquat 336 and aqueous $\mathrm{KOH}(1.0 \mathrm{mmol})$.

${ }^{\mathrm{C}}$ Considering negligible amounts of $\mathbf{4}, \mathbf{5}$, and $\mathbf{7}, \mathrm{k}_{1-5}$ cannot be determined accurately.

${ }^{\mathrm{d}}\left(\mathrm{k}_{2}{ }^{\prime}+\mathrm{k}_{3}{ }^{\prime}\right) \sim 0.05\left(\mathrm{k}_{1}{ }^{\prime}+\mathrm{k}_{4}{ }^{\prime}\right)$.

${ }^{\mathrm{e}} \mathrm{k}_{4}{ }^{\prime}$ cannot be determined accurately, and is estimated as $\mathrm{k}_{1}{ }^{\prime}<\mathrm{k}_{4}{ }^{\prime}<\mathrm{k}_{5}{ }^{\prime}$.

${ }^{f}$ A fter $80 \mathrm{~min}$.

${ }^{\mathrm{g}} \mathrm{A}$ fter $3 \mathrm{~h}$.
} 
TABLE 3

First-Order $\mathrm{R}$ ate $\mathrm{C}$ onstants for the $\mathrm{H}$ ydrogenation of A cetophenone over $5 \% \mathrm{Pt} / \mathrm{C}$ under Multiphase Conditions in the Presence of Neutral, Basic, and Various A cidic Aqueous Phases

\begin{tabular}{|c|c|c|c|c|c|c|c|c|}
\hline \multirow[b]{2}{*}{ No. } & \multirow[b]{2}{*}{ Composition of aqueous phase } & \multicolumn{7}{|c|}{ First-order constants $\times 10^{3}\left(\mathrm{~s}^{-1}\right)$} \\
\hline & & $\mathrm{k}_{1}$ & $\mathrm{k}_{2}$ & $k_{3}$ & $\mathrm{k}_{4}$ & $k_{5}$ & $k_{6}$ & $\mathrm{k}_{7}$ \\
\hline 1 & B asic medium, $\mathrm{KOH}(1.0 \mathrm{mmol})$ & $0.25 \pm 0.01$ & 0 & 0 & 0 & 0 & 0 & 0 \\
\hline 2 & Neutral, water & $0.17 \pm 0.01$ & $0.13 \pm 0.01$ & $0.05 \pm 0.01$ & $0.03 \pm 0.01$ & 0 & 0 & 0 \\
\hline 3 & Slightly acidic, $0.003 \mathrm{M} \mathrm{HCl}$ & $0.20 \pm 0.01$ & $0.12 \pm 0.01$ & $0.10 \pm 0.01$ & 0 & $(7 \pm 0.7) \times 10^{-6 a}$ & $(1 \pm 0.05) \times 10^{-7 a}$ & $-{ }^{b}$ \\
\hline 4 & A cidic, $0.118 \mathrm{M} \mathrm{HCl}$ & $0.40 \pm 0.02$ & $0.12 \pm 0.01$ & $0.16 \pm 0.02$ & 0 & $(2 \pm 0.2) \times 10^{-5 a}$ & $(3 \pm 0.1) \times 10^{-7 a}$ & $-^{\mathrm{b}}$ \\
\hline 5 & Strongly acidic, $1.18 \mathrm{M} \mathrm{HCl}$ & $1.38 \pm 0.04$ & $0.15 \pm 0.01$ & $0.13 \pm 0.01$ & 0 & $0.48 \pm 0.02$ & 0 & $0.07 \pm 0.01$ \\
\hline 6 & A cidic, ${ }^{c} 0.118 \mathrm{M} \mathrm{HCl}$ & $1.45 \pm 0.17$ & $0.45 \pm 0.13$ & $0.37 \pm 0.07$ & $0.30 \pm 0.17$ & $0.27 \pm 0.03$ & 0 & $0.60 \pm 0.15$ \\
\hline
\end{tabular}

\footnotetext{
a nitial rates ( $\mathrm{M} / \mathrm{s})$.

${ }^{\mathrm{b}} \mathrm{N}$ ot determined.

${ }^{\mathrm{c}} \mathrm{N}$ o A liquat 336.
}

of the PT agent but with $1 \% \mathrm{KOH}$, or vice versa with the PT agent (A 336) and pure water, also initially underwent dechlorination $(4,5)$.

These results can be compared to those obtained for the hydrogenation of acetophenone in the presence of $\mathrm{A}$ liquat 336 and $1 \%$ aqueous $\mathrm{KOH}$ (Table 3, entry 1 ). In the latter case no ring reduction product (7) is observed and the rate constant for the formation of $\mathbf{5}\left(\mathrm{k}_{1}\right)$ issomewhat higher. This difference probably arises from the decrease in the concentration of $\mathrm{KOH}$ (initially $1.0 \mathrm{mmol}$ ) during the reaction of p-chloroacetophenone, where it is partially neutralized by the $\mathrm{HCl}(0.7 \mathrm{mmol})$ being released.

$M$ utiphase $\mathrm{H}$ ydrogenation of $\mathrm{A}$ cetophenone in the

P resence of A cidic/B asic A queous P hase

It has recently been reported (5) for the hydrogenation of $p$-chloropropiophenone that the composition of the aqueous phase has a substantial effect on the selectivity in the catalytic multiphase reduction of different functional groups. One of the crucial factors appears to be the acidity/basicity of the aqueous phase.

With an aim to perform a detailed kinetic study of the selectivity of the reduction of other (than chlorine) functional groups, namely the carbonyl, and phenyl ring reduction and to clarify the conditions that affect the reaction kinetics and govern the selectivity, a series of experiments have been carried out. To simplify the model reaction schemes and to eliminate side effects which may arise from the removal of chlorine, all the forthcoming results have been obtained starting from the catalytic hydrogenation of acetophenone.

A number of systems, which employ neutral, basic, or various acidic compositions of aqueous phase, were probed. The rate constants for the reaction steps which occur in these systems and the corresponding conditions are reported in Table 3.

The use of pure water at $\mathrm{pH}=7$ (entry 2) affords the equally fast reduction of both carbonyl group and phenyl ring ( $4 \rightarrow(5+7) \rightarrow \mathbf{8}$ pathway), so the rate constants for these steps $\left(k_{1}\right.$ and $\left.k_{2}\right)$ are comparable. A s $\mathbf{5}$ and $\mathbf{7}$ are formed, they undergo further, but slower, reduction to $\mathbf{8}$, which is the final reduction product. A mong all the other conditions tested, the use of water, i.e., the neutral medium, results in the most favorable selectivity (nearly $40 \%$ ) toward the reduction of the phenyl ring while in most other cases it is shifted toward $\mathbf{5}$.

The presence of a basic aqueous phase ( $1 \%$ aqueous $\mathrm{KOH}$, entry 1) results in rather mild reduction. O nly the $\mathbf{4} \rightarrow \mathbf{5}$ step takes place; i.e., the selectivity is completely shifted to the reduction of the carbonyl group and $\mathbf{5}$ is the only reaction product. The rate constant for this step $\left(k_{1}\right)$ is slightly higher than that in neutral medium or in the system where $\mathrm{p}$-chloroaceto phenone is used as a substrate (Table2, entry 2).

Switching to acidic conditions (entries 3-5) the selectivity of the first step is again on the side of carbonyl reduction of the aryl ketone (while the aliphatic ketone is unreactive as explained earlier). The reaction pathway preferably follows the $\mathbf{4} \rightarrow(\mathbf{5} \rightarrow \mathbf{7}), \mathbf{5} \rightarrow(\mathbf{6}+\mathbf{8}), \mathbf{8} \rightarrow \mathbf{9}$ order in moderate acidic media and the $\mathbf{4} \rightarrow(\mathbf{5}+\mathbf{7}), \mathbf{5} \rightarrow(\mathbf{6}+\mathbf{8}), \mathbf{6} \rightarrow \mathbf{9}$ order if more acidic conditions are employed. Figure 2 displays the change in rate constants $\mathrm{k}_{1}, \mathrm{k}_{2}, \mathrm{k}_{3}, \mathrm{k}_{4}$ versus the concentration of aqueous $\mathrm{HCl}$. While the rate constants for the other steps are apparently constant and comparable, there is a sharp increase in the selectivity toward the reduction of carbonyl group with an increase in the $\mathrm{H} \mathrm{Cl}$ concentration. Increasing the acidity of the aqueous phase, there is also an increase in the yields of final reduction products, namely of ethyl benzene (6) and ethyl cyclohexane (9), especially if the strong acidic medium is employed (entry 5). This trend is in agreement with the fact that carbonyl hydrogenolysis is favored over hydrogenation in the presence of $\mathrm{HCl}$ (19).

R emarkably, the presence of aqueous acid results in the complete prohibition of the $\mathbf{7} \rightarrow \mathbf{8}$ and $\mathbf{8} \rightarrow \mathbf{9}$ steps $\left(\mathrm{k}_{4}, \mathrm{k}_{6}=0\right.$ in entries 3-5). The observed reaction pathway follows the 


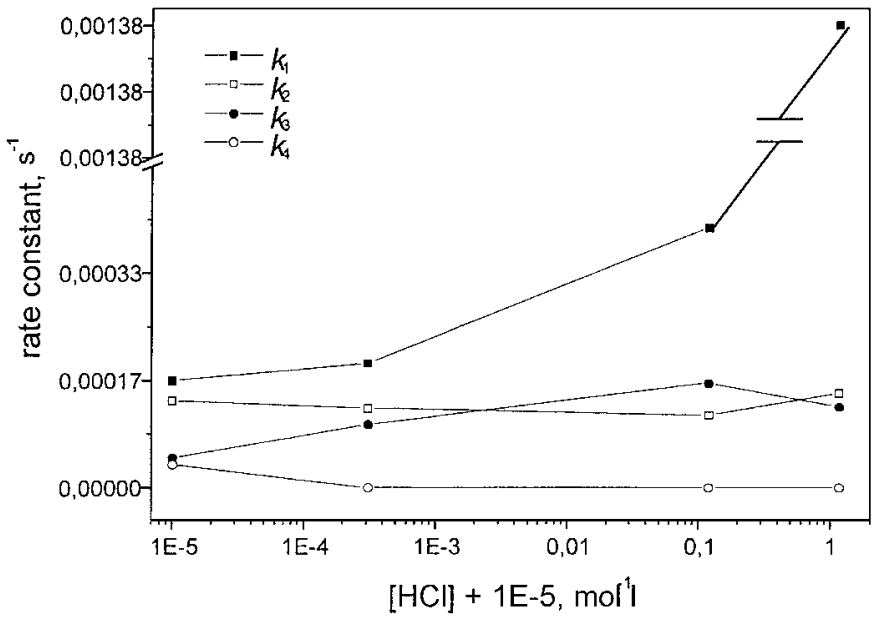

FIG . 2. E ffect of acidity on the kinetics of acetophenone hydrogenation over $5 \% \mathrm{Pt} / \mathrm{C}$ under multiphase conditions.

$\mathbf{4} \rightarrow(\mathbf{5}+\mathbf{7}) \rightarrow \mathbf{8}, \mathbf{5} \rightarrow \mathbf{6} \rightarrow \mathbf{9}$ sequence. Nevertheless, if acetophenone is reduced in acidic medium and in the absence of A liquat 336 (entry 6 ) the $\mathbf{7} \rightarrow \mathbf{8}$ step takes place, though it is quite slow and there is still no sign of the $\mathbf{8} \rightarrow$ 9 step. The same is true $\left(k_{6}=0\right)$ if $p$-chloroacetophenone is reduced in the absence of $\mathrm{A}$ liquat 336 with a neutral aqueous phase, where $\mathrm{HCl}$ is formed (Table 2 , entry 1 ). Moreover, the rate constants for the $\mathbf{6} \rightarrow \mathbf{9}$ step $\left(k_{7}\right)$ in the reduction of both $p$-chloroacetophenone (Table 2 , entry 1) and acetophenone (Table 3, entry 6) are perfectly reproduced, showing that the mathematical approach is satisfactory. The "acidic medium" behavior in the case of $p$ chloroacetophenone is due to the release of $\mathrm{H} \mathrm{Cl}$ during the HDC. The fact that the $\mathbf{7} \rightarrow \mathbf{8}$ and $\mathbf{8} \rightarrow \mathbf{9}$ steps are prohibited if A liquat 336 and an acidic environment are present can be again explained by considering the weaker adsorption of the aliphatic substrates, especially if the surface is deactivated by the presence of $\mathrm{HCl}$. The observed phenomenon seems to be a combined effect of $\mathrm{HCl}$ and A liquat 336, which cause a weaker adsorption of the aliphatic species. Indeed, if only acidic (in the absence of A liquat 336, Table 3, entry 6) conditions or if only A liquat 336 (neutral medium, Table 3, entry 2) is introduced in the reaction mixture the step corresponding to the reduction of aliphatic ketone (7) is equally prohibited. The $\mathbf{8} \rightarrow \mathbf{9}$ step is prohibited in the presence either A liquat 336 or acid, or under most other monitored conditions, but it is more likely to be prohibited due to the deactivation of the Pt catalyst over time. Presumably, the absence of PT agent produces harsher conditions for the reduction, so that the $\mathbf{7} \rightarrow \mathbf{8}$ step takes place.

\section{Influence of A queous A nions on the R eaction $\mathrm{K}$ inetics}

The additions of various inorganic salts have also been shown to affect the reaction rates. It was expected that lipophilic anions would substitute chlorine in A liquat 336 and therefore change the selectivity, since the nature of the PT agent has a major influence on the selectivity. The rate constants for the corresponding reaction steps in the systems where different salts were added are reported in Table 4.

It was found that all the tested salts can be divided into two major groups. While the additions of most salts result in more or less pronounced effects on the reaction kinetics, $\mathrm{KI}$, $\mathrm{Na}_{2} \mathrm{~S}$, and $\mathrm{KSCN}$ kill the catalyst. Only the initial rate was measured to estimate the reaction kinetics in these cases.

The majority of the other salts tested, which are listed in Table 4, also shift the selectivity of the reaction toward the

\section{TABLE 4}

First-Order R ate C onstants for the Hydrogenation of A cetophenone over 5\% Pt/C under Multiphase Conditions (A liquat 336, A queous Phase) in the Presence of Salt Additions

\begin{tabular}{|c|c|c|c|c|c|c|}
\hline \multirow{2}{*}{$\begin{array}{c}\text { Salt } \\
(0.26 \mathrm{mmol})\end{array}$} & \multicolumn{6}{|c|}{ First-order constants $\times 10^{3}\left(\mathrm{~s}^{-1}\right)$} \\
\hline & $\mathrm{k}_{1}$ & $\mathrm{k}_{2}$ & $k_{3}$ & $\mathrm{k}_{4}$ & $k_{5}$ & $\mathrm{k}_{6}$ \\
\hline $\mathrm{KCl}$ & $0.23 \pm 0.01$ & $0.16 \pm 0.01$ & $0.07 \pm 0.00$ & $0.02 \pm 0.00$ & 0 & 0 \\
\hline $\mathrm{KBr}^{\mathrm{a}}$ & $0.19 \pm 0.01$ & $0.15 \pm 0.01$ & $0.10 \pm 0.01$ & 0 & 0 & 0 \\
\hline $\mathrm{KBr}^{\mathrm{b}}$ & $0.42 \pm 0.02$ & $0.14 \pm 0.01$ & $0.08 \pm 0.00$ & 0 & $4 \times 10^{-5} \pm 2 \times 10^{-6 d}$ & $2 \times 10^{-5} \pm 3 \times 10^{-7 d}$ \\
\hline $\mathrm{KI}$ & $7 \times 10^{-7} \pm 5 \times 10^{-8 d}$ & $3 \times 10^{-7} \pm 2 \times 10^{-8 d}$ & $1 \times 10^{-7}$ & $1 \times 10^{-8 d}$ & & \\
\hline $\mathrm{Na}_{2} \mathrm{~S}$ & $2 \times 10^{-7} \pm 3 \times 10^{-8 d}$ & $7 \times 10^{-8} \pm 6 \times 10^{-9 d}$ & $1 \times 10^{-7}$ & $5 \times 10^{-9 d}$ & & \\
\hline $\mathrm{KSCN}$ & $3 \times 10^{-7} \pm 8 \times 10^{-8 d}$ & $7 \times 10^{-8} \pm 5 \times 10^{-9 d}$ & $1 \times 10^{-7}$ & $1 \times 10^{-9 d}$ & & \\
\hline $\mathrm{CH}_{3} \mathrm{COONa}$ & $0.12 \pm 0.01$ & $0.07 \pm 0.00$ & $0.02 \pm 0.00$ & $0.06 \pm 0.01$ & $5 \times 10^{-8} \pm 6 \times 10^{-9 d}$ & $8 \times 10^{-8} \pm 3 \times 10^{-9 d}$ \\
\hline $\mathrm{KHSO}_{4}$ & $0.37 \pm 0.02$ & $0.17 \pm 0.01$ & $0.09 \pm 0.01$ & 0 & $3 \times 10^{-7} \pm 3 \times 10^{-8 d}$ & $3 \times 10^{-7} \pm 1 \times 10^{-8 d}$ \\
\hline $\mathrm{NaSO}_{4} \mathrm{C}_{12} \mathrm{H}_{23}$ & $0.33 \pm 0.02$ & $0.12 \pm 0.02$ & $0.09 \pm 0.01$ & $0.10 \pm 0.01$ & $1 \times 10^{-7} \pm 8 \times 10^{-9 d}$ & $1 \times 10^{-7} \pm 5 \times 10^{-9 d}$ \\
\hline $\mathrm{NaSO}_{4} \mathrm{C}_{12} \mathrm{H}_{23}{ }^{\mathrm{c}}$ & $0.84 \pm 0.07$ & $0.20 \pm 0.04$ & $0.17 \pm 0.01$ & $0.04 \pm 0.02$ & $3 \times 10^{-7} \pm 2 \times 10^{-8 d}$ & $3 \times 10^{-7} \pm 6 \times 10^{-9 d}$ \\
\hline
\end{tabular}

a $0.7 \mathrm{mmol}$ of salt.

${ }^{b} 0.7 \mathrm{mmol}$ of salt in the presence of aqueous $\mathrm{HCl}(0.118 \mathrm{M})$.

${ }^{c}$ In the absence of A liquat 336.

${ }^{\mathrm{d}}$ Initial rates (M/s). 


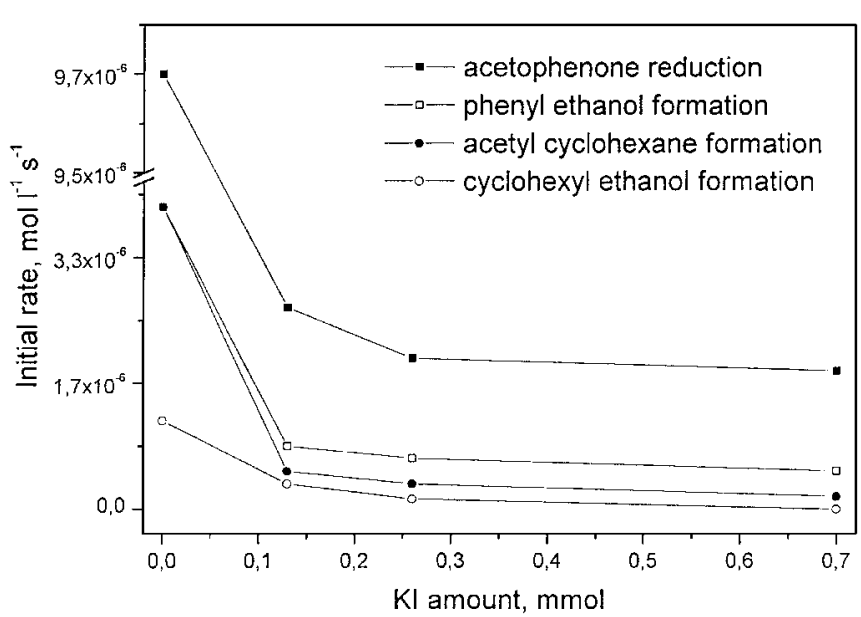

FIG . 3. E ffect of $\mathrm{KI}$ aqueous concentration on the initial rates in the multiphase hydrogenation of acetophenone over $5 \% \mathrm{Pt} / \mathrm{C}$.

reduction of the carbonyl group. Compared to the blank experiment, where no salt is added (pure water as the aqueous phase, entry 2 in Table 3 ), this effect is more or less pronounced from one salt to another and results mainly from the acceleration of $\mathrm{k}_{1}$, while $\mathrm{k}_{2}$ and $\mathrm{k}_{3}$ apparently stay constant. The exception is $\mathrm{CH}_{3} \mathrm{COONa}$, which results in a two-fold decrease in the rate constants for all the reaction steps comparing to the blank reaction, but $k_{1}$ still prevails over $k_{2}$. M oreover, in the presence of some salts the overall reduction is deeper, so that the higher yields of the final reduction products ( 6 and $\mathbf{9}$ ) are observed.

A dditions of salts such as $\mathrm{K} \mathrm{HSO}_{4}$ or $\mathrm{K} \mathrm{Br}$ have an effect similar to that in the acidic media, namely the complete inhibition of the $\mathbf{7} \rightarrow \mathbf{8}$ step $\left(\mathrm{k}_{4}=0\right)$. This is understandable for $\mathrm{KHSO}_{4}$, which is acidic itself, but is quite surprising for $\mathrm{KBr}$. The use of $\mathrm{KBr}$ in the presence of aqueous acid leads to a further increase in $k_{1}$ (it is the same as without $\mathrm{K} \mathrm{Br}$, but only acid is added), while all the other rate constants are not affected.
O ne of the most interesting effects observed is the substantial acceleration of the reaction rates in the presence of a long-chain lipophilic anion (dodecylsulfate), especially if it is used in the absence of A liquat 336. Probably, such salts, which manifest the properties of surfactants, may produce a co-catalyzing micellar effect by modifying the surface of a supported Pt catalyst. This effect does not seem to be the same as the one produced by the PT agent, because A liquat 336 does not usually speed up the reaction, but rather softens the conditions affording the yields of less reduced products. The effect of surfactant in this case affords a faster reduction at all steps, not much affecting the selectivity. Seemingly, this effect is suppressed if PT agent is already sorbed on the surface.

\section{$\mathrm{H}$ ydrogenation of $\mathrm{A}$ cetophenone under $\mathrm{O}$ ther $\mathrm{C}$ onditions}

To extend the scope of the monitored conditions, which may affect the behavior of the catalytic hydrogenation, and to elucidate the peculiarities of the multiphase systems, a series of experiments set up in alternative systems has also been carried out. The results on the rate constants for these systems are reported in Table 5.

A s was previously mentioned, the absence of A liquat 336 results in much harsher reduction conditions. U nder these conditions, much higher rate constants for all the reaction steps are observed, especially for the formation of the final reduction products $(\mathbf{6}, \mathbf{9})$. The selectivity of the first step is shifted to the formation of $\mathbf{5}$.

In the absence of aqueous phase, there is no substantial difference in the rate constants compared to the those of blank reaction (Table 3 , entry 2 ). The selectivity on the first step is slightly shifted to the formation of $\mathbf{5}$, and like in acidic media, the reduction of acetyl cyclohexane (7) is suppressed. There are also small amounts of final reduction products ( 6 and 9 ) formed.

A two-fold decrease in the amount of $A$ liquat 336 brings no essential changes to the reaction kinetics, though it

\section{TABLE 5}

\section{First-Order R ate C onstants for the Hydrogenation of A cetophenone in Various C atalytic Systems}

Conditions

\begin{tabular}{|c|c|c|c|c|c|c|c|c|c|c|}
\hline No. & Catalyst & A q. phase & PT agent & Solvent & $\mathrm{k}_{1}$ & $\mathrm{k}_{2}$ & $\mathrm{k}_{3}$ & $\mathrm{k}_{4}$ & $k_{5}$ & $\mathrm{k}_{6}$ \\
\hline 1 & $5 \% \mathrm{Pt} / \mathrm{C}$ & Water & None & I sooctane & $0.64 \pm 0.01$ & $0.24 \pm 0.09$ & $0.42 \pm 0.10$ & $0.34 \pm 0.22$ & $0.14 \pm 0.03$ & $0.53=$ \\
\hline 2 & $5 \% \mathrm{Pt} / \mathrm{C}$ & None & A liquat & I sooctane & $0.26 \pm 0.01$ & $0.13 \pm 0.01$ & $0.08 \pm 0.01$ & 0 & $2 \times 10^{-7} \pm 3 \times 10^{-8 c}$ & $1 \times 10^{-7} \pm 6 \times 10^{-9 c}$ \\
\hline 3 & $5 \% \mathrm{Pt} / \mathrm{C}$ & Water & A liquat ${ }^{a}$ & I sooctane & $0.17 \pm 0.01$ & $0.14 \pm 0.01$ & $0.08 \pm 0.01$ & $0.02 \pm 0.00$ & $1 \times 10^{-7} \pm 8 \times 10^{-9 c}$ & $1 \times 10^{-7} \pm 1 \times 10^{-9 c}$ \\
\hline 4 & $5 \%{ }^{b} \mathrm{Pt} / \mathrm{C}$ & Water & A liquat & I sooctane & $0.06 \pm 0.00$ & $0.04 \pm 0.00$ & $0.02 \pm 0.00$ & $0.02 \pm 0.01$ & 0 & 0 \\
\hline 5 & $5 \% \mathrm{Pt} / \mathrm{C}$ & None & None & E thanol & $0.52 \pm 0.02$ & $0.08 \pm 0.01$ & $0.02 \pm 0.00$ & 0 & $8 \times 10^{-7} \pm 1 \times 10^{-8 c}$ & $2 \times 10^{-7} \pm 1 \times 10^{-8 c}$ \\
\hline 6 & $10 \% \mathrm{Pd} / \mathrm{C}$ & Water & A liquat & I sooctane & $0.68 \pm 0.05$ & $0.02 \pm 0.00$ & 0 & 0 & 0 & 0 \\
\hline
\end{tabular}

\footnotetext{
${ }^{\mathrm{a}} \mathrm{H}$ alved amount of $\mathrm{A}$ liquat 336 is used $(0.13 \mathrm{mmol})$.

${ }^{\mathrm{b}} \mathrm{H}$ alved amount of $\mathrm{Pt} / \mathrm{C}$ is used $(0.0419 \mathrm{~g})$.

${ }^{\mathrm{c}}$ Initial rates ( $\left.\mathrm{M} / \mathrm{s}\right)$.

${ }^{d} k_{7}$.
} 
causes some reduction toward the final hydrogenation products. N evertheless, even this halved amount of A liquat 336 produces substantial changes compared to the reaction with no PT agent. Probably, there is saturation on the amount of $A$ liquat 336 , which is required to produce the effect of multiphase conditions.

R emarkably, a two-fold decrease in the amount of supported Pt catalyst leads to the corresponding decrease in all the reaction rates, not much affecting the selectivity. This testifies that only the supported metal catalyst contributes to the actual catalytic effect, and the hydrogenation reaction is not possible in the absence of it.

Under otherwise similar conditions, $10 \% \mathrm{Pd} / \mathrm{C}$ sharply differs from $5 \% \mathrm{Pt} / \mathrm{C}$, resulting in a much milder (not deep) reduction. The use of the $\mathrm{Pd}$ catalyst resemblesthe behavior of the Pt catalyst in the presence of aqueous base, where only one-step reduction takes place with selectivity strongly shifted to the reduction of the carbonyl group.

The reaction in ethanol in the absence of $A$ liquat 336 and aqueous phase constitutes the usual heterogeneous catalytic hydrogenation. The rate constants for most steps are rather low except for a high value for $k_{1}$. R emarkably, the "acidic-like" effect takes place again in this case $\left(\mathrm{k}_{4}=0\right)$.

\section{CONCLUSIONS}

In this work, a kinetic study has been carried out to estimate the rates and the selectivities and to determine the effects of different reaction conditions on the selectivity in this multiphase catalytic hydrogenation system. A kinetic approach and a number of mathematical rate expressions are derived and successfully applied for the estimation of rate constants at each reaction step. The possible effect of the reagent/intermediate adsorption over the supported catalyst does not much affect the observed first-order kinetics and so the adsorption corrections were not included in the observed rate constants.

It has been shown that the use of multiphase conditions, namely the use of a PT agent coupled with a supported metal catalyst in the presence of aqueous phase, results in the selective acceleration of the chlorine removal as well as in softer reduction conditions, preventing the formation of the final reduction products and giving substantial yields of either aromatic ring or carbonyl reduction products. The selectivity in the first step can be controlled to some extent and is mostly shifted to the reduction of the carbonyl group. The most favorable selectivity toward reduction of phenyl ring is observed if no $\mathrm{KOH}$ is used as an aqueous phase in the presence of $\mathrm{A}$ liquat 336. The completely selective reduction of carbonyl occurs in the presence of aqueous base or if $\mathrm{Pd}$ catalysts are used.

The effects of basic and acidic aqueous media have been investigated. The use of base results in the selective reaction toward the carbonyl group reduction, whereas in acidic media the reduction conditions are harsher so the reaction proceedstoward the formation of completely reduced products. M oreover, the acidic multiphase conditions prohibit the reduction of cyclohexyl derivatives.

It has also been found that additions of inorganic salts may have a substantial impact on the reaction kinetics. Salts such as iodides, sulfides, or thiocyanides inhibit the reduction by poisoning the surface of the supported metal catalyst, while the majority of other inorganic salts do not exert any pronounced influence. R emarkably, in the presence of surfactants such as sodium laurylsulfate the reaction is significantly accelerated, especially if no PT agent is used. This reveals new possibilities to modify the multiphase conditions by coupling them with a co-catalyzing micellar effect.

\section{ACKNOWLEDGMENTS}

This work was supported by I NCA (I nteruniversity Consortium Chemistry for the E nvironment) and M U R ST (M inistero U niversitá e R icerca Scientifica e Technologica) fondo $60 \%$.

\section{REFERENCES}

1. M arques, C. A ., Selva, M., and Tundo, P., J. Chem. Soc., Perkin Trans. 1529 (1993); M arques, C. A ., Selva, M., and Tundo, P., J. O rg. Chem. 58, 5256 (1993); M arques, C. A ., R ogozhnikova, O., Selva, M., and Tundo, P., J. M ol. Catal. A : Chemical 96, 301 (1995).

2. Marques, C. A., Selva, M., and Tundo, P., J. Org. Chem. 59, 3830 (1994).

3. Marques, C. A., Selva, M., and Tundo, P., J. Org. Chem. 60, 2430 (1995).

4. Selva, M., Tundo, P., and Perosa, A ., J. O rg. Chem. 63, 3266 (1998).

5. Perosa, A., Selva, M., and Tundo, P., J. Org. Chem. 64, 3934 (1999).

6. Okano, T., Tsukiyama, K., and Konishi, H., Chem. L ett., 603-606 (1982).

7. Hoke, J.H., Gramiccioni, G.A., and Balko, E. N., A ppl. Catal: B 1, 285-296 (1992).

8. Shin, E.-J., and K aane, M. A., Catal. L ett. 58, 141-145 (1999).

9. La Pierre, R. B., Wu, D., K ranich, W. L., and Weiss, A. H., J. Catal. 52, 59-71 (1978).

10. Pasett, P., and G entile, F., J. O rg. Chem. 60, 1351 (1995).

11. O kano, T., Tsukiyama, K., Konishi, H., and K iji, J., Chem. L ett., 603 (1982); Tung, H.-S., B rubaker, C. H., J r., J. O rganomet. Chem. 216, 129 (1981).

12. Bomben, A., Marques, C.A., Selva, M., and Tundo, P., Synthesis 1109 (1996).

13. M usoke, J., Roberts, D., and Cooke, M., Bull. Environ. Contam. Toxicol. 28, 467 (1982).

14. "Pesticides D isposal and D etoxification: Processes and Techniques" (A. P. D illon, E d.), N oyes D ata Corp., Parkridge, NJ, 1981.

15. Hagh, B. F., and Allen, D. T., in "Innovative Hazardous Waste Treatment Technology Series" (H. M . Freeman, E d.), Vol. 2, Physical/ Chemical Processes, pp. 45-53. Technomic Publ. Inc., L ancaster, PA, 1990.

16. Kalnes, T. N., and Jemmes, R. B., in "Innovative H azardous Waste Treatment Technology Series" (H. M . Freeman, E d.), Vol. 1, Thermal Processes, pp. 65-76. Technomic Publ. Inc., L ancaster, PA, 1990.

17. Beranek, L., in "A dvances in Catalysis," Vol. 24, pp. 1-55. A cademic Press, San Diego, CA, 1975.

18. Schmid, R., and Sapunov, V. N., "Non-Formal Kinetics." Verlag Chemie $\mathrm{G}$ mbH , Weinheim, 1982.

19. A rmandia, M.A., B orau, V., Jimenez, C., M arinas, J. M., Sempere, M. E., and U rbano, P., A ppl. Catal. 43, 47 (1988). 